\title{
Mutaciones Planetarias. Oikos Nomos de la Información ¿Un Nuevo Orden Tecnológico Mundial?
}

\author{
Gustavo Arce ${ }^{(*)}$
}

\begin{abstract}
Resumen: Desde la perspectiva de la Economía Política de las relaciones económicas internacionales, este trabajo describe y analiza la emergente estructura de la economía mundial, basada, fundamentalmente, en el Poder que surge de quienes producen, comercian y controlan el saber y el conocimiento. En el último tercio del siglo XX, los núcleos capitalistas más poderosos - Estados, Empresas globales y la "tecnociencia" - , tanto del Nor te como del Sur, inauguraron un progresivo, desigual, inexorable y revolucionario proceso de mutación en las condiciones donde se produce "la mercancía compleja" capitalista. Hacia 1970, la simbiosis entre la producción de la ciencia y la producción de bienes económicos o de mercancías, marcó el ingreso del sistema capitalista y de la Humanidad a la Economía de la Información y a la Sociedad del Conocimiento. La magnitud de la mutación técnica, social, económica y política del capitalismo "tecno-informacional" explica las alteraciones que sufren las relaciones del hombre con la Naturaleza y la Vida, de los Estados y de las Naciones en sus conflictivas relaciones en el seno del Sistema Internacional. Desde la perspectiva de la Economía Política de las relaciones económicas internacionales, este trabajo describe y analiza la emergente estructura de la economía mundial, basada, fundamentalmente, en el Poder que surge de quienes producen, comercian y controlan el saber y el conocimiento.
\end{abstract}

Palabras-clave: Crisis Mundial, Crisis Taylorista/Fordista, Revolución Científica, Economía de la Información, Nueva Economía.

\begin{abstract}
In the perspective of the International Political Economy this paper describes and analyzes the new world economy structure, based essentially, on the power of the capitalist centers that produce, trade and control the knowledge, and the information. In the last third of the XXth century the more powerful capitalist centers - States, Global Enterprises and the Technoscience wherever, in the North or/and in the South, started a progressive, unequal, inexorable and revolutionary process of changes on the environment where the capitalist complex merchandise is produced. Around 1979, the symbiosis between production and science in the productive processes of goods marked the beginning of the capitalist system in the Economy of Information or the Knowledge Society. The extent of the mutation in technology, society, economy and politics explains changes in the relations between man, nature and life, status and nations and the nations and their conflictive relations in the international system.
\end{abstract}

Keywords: Global Crisis, Toylorist/Fordist Crisis, Scientific Revolution, Information Economy, Information Society, New Economy.

(*) Licencia, Maestría y Doctorado en Economía - Universidad de París 8/Francia, Catedrático de Economía Política y de Economía I y II, Coordinador de la Licenciatura en Relaciones Internacionales, Coordinador de la Sala Docente de Economía — Facultad de Derecho — UdelaR. E-mail: arpu@adinet.com.uy. Recebido em 8.6.09 e aceito em 25.6.09. 


\section{INTRODUCCIÓN}

\section{Mutaciones Planetarias 1: Del bloqueo generalizado de la lógica TAYLORISTA-FORDISTA A LA RELACIÓN SOCIAL DE SERVICIO. LA NUEVA MATRIZ PRODUCTIVA DEL CAPITALISMO DE LA INFORMACIÓN}

En el último tercio del siglo XX, el crecimiento económico se detiene en las dos economías líderes que ordenaban, entonces, la economía mundial ${ }^{(1)}$ y las relaciones internacionales: los EEUU y la URSS. En la Unión Soviética, y su zona de influencia, la crisis económica se expresó en una caída de alrededor de un 40\% de la producción industrial, con descensos notorios en la productividad del trabajo y en el rendimiento del capital invertido (GANBEGUIAN, 1987). Del bloqueo y agotamiento de su modelo de desarrollo económico, basado en los principios de la división taylorista del trabajo, en la década de los años 80, la URSS conocerá en la década siguiente su crisis política que desembocará en su auto-disolución económica y política, a pesar de los intentos que para evitarla significaron la Perestroika y la Glasnot.

En los EEUU, en Japón y en las economías líderes de la entonces Comunidad Económica Europea (Alemania, Francia, Reino Unido), la lógica taylor-fordista, que animó las "treinta gloriosas", comenzó a manifestar signos de su agotamiento, con descensos en el ritmo de variación del producto y de la demanda interna, y el desarrollo de la llamada "estanflación".

A diferencia de lo que le sucedió a la URSS, la crisis en el Occidente capitalista desarrollado no conllevó un cambio político/social de la magnitud que sacudió a la lógica estatal de producción reinante en el este, sino a una mutación de y en las condiciones donde se produce la mercancía capitalista.

Dicha simbiosis engendró y puso en marcha, un violento proceso de mutación técnico, científico, social, político y cultural, no sólo del sistema capitalista, quien ingresó, entonces, en su fase post-industrial, de economía inmaterial, de la información y del conocimiento (ARCE, 2001. p. 9-33), sino también, de la Humanidad que inició una nueva era en su ya milenaria existencia.

La posibilidad de aprehender, descifrar y manipular la información que contiene la Materia, le permitió al Hombre, y sobre todo, a aquellos que producen, controlan o difunden el Saber, un Poder sobre las leyes del Universo, que hasta entonces, pertenecía a los Dioses, a las leyes de la Naturaleza, a la Fatalidad o a un Dios, casi en exclusividad. La capacidad de penetrar en lo más íntimo de la Vida y por ende en su propia creación y manipulación, indica y explica las novedosas, profundas y revolucionarias modificaciones en las relaciones del Hombre con la Naturaleza, noveles teorías sobre la Materia y la Energía, profundización de las leyes de Newton, cuestionamiento de los fundamentos filosóficos y políticos del Siglo de las Luces.

(1) Definida como el conjunto de relaciones económicas (productivas, comerciales, financieras, tecnológicas, materiales e inmateriales, lícitas e ilícitas, que se desarrollan a escala planetaria y que son animadas y estructuradas por la lógica de producción capitalista). 
En consecuencia, una mutación civilizacional con impactos y efectos sobre las reglas que vinculan al Hombre con la Naturaleza y la Vida, a las empresas y a las organizaciones, a los estados y a las naciones en el sistema internacional; una mutación similar, por ejemplo, a la que sucedió cuando la Humanidad ingresó sea a la Era del Neolítico o a la Era Moderna.

Por estos y muchos otros argumentos (que no tenemos aquí el espacio para desarrollarlos), en el último tercio del siglo XX, asistimos a una mutación civilizacional en la Historia de la Humanidad y a un cambio cualitativo en la estructura y en el funcionamiento de la producción capitalista. En fin, un Nuevo Mundo como en el siglo XV. Como en aquel, en éste, el capitalismo también produce Riqueza, aunque ella asume formas y modalidades un poco diferentes a las de entonces. Ahora, casi todo acto humano forma parte del Reino de la mercancía y de la lógica del beneficio. Los monopolios, públicos y/o privados, mixtos, nacionales y/o multinacionales, como sus ancestros de los siglos XIV, XV y XVI, compiten desenfrenadamente a escala planetaria, donde libran una feroz guerra comercial, para conquistar y/o preservar su lugar en el Nuevo Mundo y en la "Nueva" Economía.

\section{Mutación PlanetaRia Y OIKOS NOMOS DE LA INFORMACIÓN}

La posibilidad de aprehender la Materia gracias a la información que ella contiene, permite descifrar datos utilizando un mínimo de Energía. La informatización de los procesos productivos (materiales e inmateriales) revoluciona la oferta y modifica los cimientos de la división social y técnica del trabajo, teorizada desde Aristóteles hasta Emilio Durkheim. A diferencia del modelo taylor-fordista, pensado y estructurado desde la producción (la oferta) hacia el mercado, el método inventado por el ingeniero japonés Taiichi Ohno, la producción se organiza desde el mercado (la demanda) hacia la producción y ello simboliza, nítidamente, la nueva arquitectura de los procesos productivos que engendra y posibilita el manejo del conocimiento y la información.

En efecto, la posibilidad de poseer el conocimiento y el saber ex ante de la producción de las mercancías está revolucionando el proceso productivo capitalista, puesto que ahora se producirá lo que el consumidor "quiera" y "pueda" comprar. En la economía de la información y en la sociedad del conocimiento, el capitalismo tiende, de más en más, a producir (en tiempo real y "on line") lo que ya previamente vendió. El método Ohno, a diferencia del taylorista, al organizar el trabajo, desde la demanda hacia la oferta, permite que se pueda lograr nuevos aumentos de productividad (del trabajo vivo y del capital), por procedimientos que eliminan tanto los tiempos muertos como los gestos inútiles de los trabajadores. Esto se conoce popularmente como el método de los cinco ceros: la empresa reduce o elimina el stock (primer cero); reduce o elimina los defectos en la producción de los bienes (segundo cero); reduce o elimina las probables averías de los bienes (tercer cero); reduce o elimina la burocracia propia al sistema compartimentado entre los diferentes servicios de una empresa taylorista (cuarto cero); finalmente la entrega del producto al consumidor en tiempo real (quinto cero). El método Ohno se materializa en los llamados círculos de calidad de los trabajadores, lo que implica la polivalencia y la flexibilidad de los mismos, en el proceso de trabajo. En consecuencia, a diferencia del taylorismo, el ohnismo reintroduce la inteligencia y la creatividad del obrero en la producción de los bienes. 
La progresiva y exitosa introducción del ohnismo en los más variados procesos productivos - desde el fast food: Mc Donald's, a la producción del conocimiento y del saber - no implicó la desaparición del método taylorista de producción. La generalización del método Ohno exige una profundización del método taylorista de la división del trabajo. Éste se aplicará no solo en los procesos industriales, sino ahora, asociado al método Ohno, el taylorismo ingresará en la esfera inmaterial de la producción — la oficina administrativa, el laboratorio de investigación científica, en los sistemas de salud, la enseñanza, los medios de comunicación, la producción de contenidos audiovisuales y de multimedia ${ }^{(2)}$.

Esta transformación en la matriz productiva, es uno de los principales indicadores de la mutación del capitalismo y del cambio de civilización propio de la economía mundial y del sistema internacional del siglo XXI.

La mutación en la forma misma de producir la mercancía compleja ${ }^{(3)}$ se manifiesta claramente (al menos) por cuatro razones: a. la producción en flujos; b. la producción/ control de la información (producir el Conocimiento); c. la inversión inmaterial y los activos inmateriales $(\mathrm{I}+\mathrm{D})$; $\mathbf{d}$. la constitución de densas redes telemáticas (ciberespacio), en donde circula la información y en las cuales los usuarios acceden en un tiempo determinado y mediante el pago de un precio, fundamentalmente a través de contratos de adhesión.

En el capitalismo de la información y en la sociedad del conocimiento, las mutaciones en la estructura productiva heredada de la primera y segunda revolución industrial (taylorismo y sus diferentes versiones) se plasman en una nueva matriz (Ohno-taylorista) que, a su vez, cobra realidad técnica, económica y social, en una nueva relación social de producción: la Relación Social de Servicio.

Es una relación social de producción que vincula tres actores: un demandante/ consumidor/usuario (B) contrata los servicios de un oferente/prestatario (A), para realizar la transformación de una realidad/objeto (C), en su beneficio.

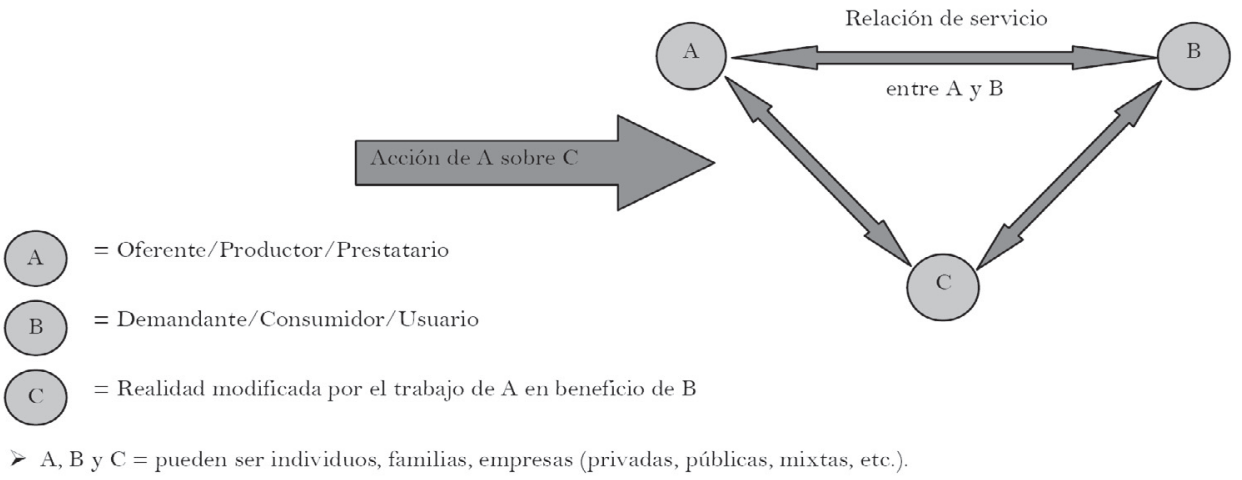

Fuente: GADREY, Jean: L'économie des services. Paris: La Découverte, Collection Repères, 1996. p. 19.

(2) Sobre estas transformaciones se puede leer, entre otros autores, Rifkin (2000) — existe versión en español, Paidós, Duval (1998).

(3) Véase, Beaud (1997. p. 196).

114 
Independientemente de la escala que se analice - micro/macro, local/nacional/ internacional - o, del sector de actividad estudiado - material (agrícola, industrial), e inmaterial (seguridad, defensa, limpieza, justicia, enseñanza, salud, multimedia, etc.) — la relación social de servicio reorganiza la clásica relación bilateral y contractual de intercambio en el mercado, forjada por los pensadores griegos y retomada por los clásicos de la economía política de los siglos XVIII y XIX. La trilateralidad, propia a la relación social de servicio, vuelve a dividir y a modificar las bases organizacionales de los procesos de producción afectando tanto a las relaciones entre las empresas: subcontratación/tercerización/flexibilización, como de éstas con los trabajadores: subcontratación/tercerización/flexibilización ${ }^{(4)}$.

La instauración de una nueva matriz productiva y el establecimiento de la relación social de servicio, expresan claramente el ingreso y la reproducción del capitalismo bajo las reglas propias al oikonomos de la información.

Otra manifestación tan importante como incontrovertible - aunque no la única -, de la mutación capitalista en curso, es la reconfiguración y el desplazamiento de los centros de poder en el espacio económico mundial. En las próximas tres o cuatro décadas, la economía mundial se asentará definitivamente sobre una bipolaridad geoeconómica compartida entre el polo Unión Europea/ USA y el polo Asia. Entre esos dos polos, el producto mundial se repartirá casi en partes iguales ( $41 \%$ y $45 \%$ respectivamente); en cuanto a la población mundial, casi el 40\% de la misma se concentrará en China e India, economías y Estados que reaparecen en la escena internacional de donde el Occidente capitalista las desplazó alrededor del 1600.

\begin{tabular}{|l|c|c|c|}
\hline \multicolumn{4}{|c|}{$\begin{array}{c}\text { LA DISTRIBUCIÓN MUNDIAL DEL PRODUCTO } \\
\text { EN GRANDES REGIONES } \\
\text { (en porcentajes) }\end{array}$} \\
\hline ZONA/PAÍSES & $\mathbf{2 0 0 4}$ & $\mathbf{2 0 2 5}$ & $\mathbf{2 0 5 0}$ \\
\hline Unión Europea & 34 & 25 & 15 \\
\hline USA & 28 & 27 & 26 \\
\hline Japón & 12 & 7 & 4 \\
\hline China & 4 & 15 & 28 \\
\hline India & 2 & 6 & 17 \\
\hline Otros & 20 & 20 & 10 \\
\hline
\end{tabular}

Fuente: Tomado de Bilan du Monde 2007. L'Atlas de 174 pays. Paris: Le Monde, 2007. p. 12.

(4) Ejemplo de las mutaciones propias a la relación social de servicio y su impacto sobre las relaciones laborales, para el caso uruguayo se puede leer, Rosembaum y Castello (2007). 
Gustavo Arce - Cadernos PROLAM/USP (Ano 8 - Vol. 1 - 2009) p. 111-123

\begin{tabular}{|c|c|c|c|c|c|}
\hline \multicolumn{6}{|c|}{$\begin{array}{l}\text { LOS ONCE ESTADOS MÁS POBLADOS EN EL MUNDO } \\
\text { (en millones de personas) }\end{array}$} \\
\hline Países & Año 2001 & $\begin{array}{c}\text { \% sobre } \\
\text { Total } \\
\text { Mundial }\end{array}$ & Países & $\begin{array}{c}\text { Proyección } \\
\text { Año } 2015\end{array}$ & $\begin{array}{c}\text { \% sobre } \\
\text { Total } \\
\text { Mundial }\end{array}$ \\
\hline China & $1.285,2$ & 21,0 & China & $1.402,3$ & 19,5 \\
\hline India & $1.033,4$ & 16,8 & India & $1.246,4$ & 17,3 \\
\hline EEUU & 288,0 & 4,7 & EEUU & 329,7 & 4,6 \\
\hline Indonesia & 214,4 & 3,5 & Indonesia & 250,4 & 3,5 \\
\hline Brasil & 174,0 & 2,9 & Pakistán & 204,5 & 2,8 \\
\hline Pakistán & 146,3 & 2,4 & Brasil & 202,0 & 2,8 \\
\hline Rusia & 144,9 & 2,4 & Bangladesh & 181,4 & 2,5 \\
\hline Bangladesh & 140,9 & 2,3 & Nigeria & 161,7 & 2,2 \\
\hline Japón & 127,3 & 2,1 & Rusia & 133,4 & 1,9 \\
\hline Nigeria & 117,8 & 2,0 & Japón & 127,2 & 1,8 \\
\hline México & 100,5 & 1,6 & México & 119,6 & 1,7 \\
\hline $\begin{array}{r}\text { Total } \\
\text { Mundial }\end{array}$ & $6.148,1$ & $\cong 62,3$ & $\begin{array}{r}\text { Total } \\
\text { Mundial } \\
\end{array}$ & $7.197,2$ & $\cong 60,6$ \\
\hline
\end{tabular}

Fuente: Elaboración propia con datos de PNUD. Informe sobre desarrollo humano 2003. Cuadro 5. p. 250 a 253

\section{Mutaciones Planetarias 2: ¿ ¿UN NUEVO ORDEN TECNOLÓGico De la ECONOMía MUNDIAL DEL SIGLO XXI?}

En el capitalismo de la información, la Riqueza - sea ella considerada a nivel micro o macro - consiste en la transformación de toda Idea, Saber, o Conocimiento - industrial o artístico - en un bien económico o en una mercancía intercambiable en el mercado o con posibilidades de acceder a ella en el ciberespacio.

Los indicadores más comúnmente utilizados para medir la capacidad creativa de las energías del pensamiento humano, son:

- para la creación de conocimiento, los gastos en Investigación y Desarrollo (I+D) como parte de la totalidad de los bienes de usos final, producidos en un sector de actividad específico, o, en la totalidad de un sistema económico (PBI); y/o la cantidad de investigadores en $\mathrm{I}+\mathrm{D}$ por cada millón de personas, y/o la cantidad de patentes otorgadas a residentes sobre millón de personas, y/o la recaudación por concepto de royalties y derechos de explotación por habitante.

- para la difusión de conocimiento, los indicadores más frecuentemente utilizados son aquellos que miden el uso y el usufructo de un bien económico o de una mercancía, fruto de la innovación científica y técnica por un número cada vez mayor de usuarios (personas físicas o jurídicas, públicas o privadas, mixtas, etc.), como por ejemplo lo es la cantidad de teléfonos celulares, o de productos informáticos, o de usuarios de internet, por cada mil de habitantes.

Una de las fuentes más exhaustivas del punto de vista estadístico, sobre el tema que nos ocupa, es la que produce y publica la Organización de Cooperación y Desarrollo 
Económico (OCDE), consagrada a la presentación y el análisis de los Principales Indicadores de la Ciencia y de la Tecnología. Para el presente trabajo, hemos utilizado el Informe del Observatoire des Sciences et des Techniques(5) (OST). El mismo, no sólo recoge los datos de la OCDE, sino también los de los otros trece miembros del Observatorio, Ministerios, Institutos de Investigación, Universidades, Grandes Escuelas, tanto franceses como europeos.

La muestra del Informe OST comprende a los treinta Estados miembros OCDE más la llamada OCDE "plus"(6), es decir, aquellos Estados que en el campo de la I+D han sido asociados a los treinta miembros de la OCDE. Por lo tanto, el análisis sobre el poder tecnológico actual se realiza a partir de ese grupo de Estados que representan el $14 \%$ de la población mundial, pero que concentran casi las 3/4 partes del ingreso bruto mundial, el $84 \%$ del comercio mundial y el $60 \%$ del consumo mundial de energía.

En cuanto a las empresas, la muestra utilizada censó 10.500 empresas, de las cuales 4.100 son consideradas como empresas con fuerte componente en $\mathrm{I}+\mathrm{D}$, pues invierten anualmente en ese rubro, una cifra cercana a 1,5 millón de euros.

Según el Informe de la OST, la principal característica de la producción de la ciencia y de la tecnología, es su alta concentración oligopólica, verificable tanto:

- por región geográfica,

- por Estados miembros de las regiones,

- por grandes sectores de la actividad económica,

- por su financiamiento (principalmente privado y con fines civiles, en la Unión Europea y Japón, mientras que el destino militar prima en los Estados Unidos),

- como en el ámbito de las capacidades científicas (investigadores a tiempo completo, cantidad de estudiantes de nivel superior, cantidad de doctores, la producción tecnológica medida en cantidad de patentes y de publicaciones científicas).

El Informe registra e investiga fundamentalmente la producción del Saber en la Economía Industrial ${ }^{(7)}$; la unidad de medida es el millardo de euros en paridad de poder de compra y el período analizado es la década 1993-2003.

\section{LA MAGNITUd Y LA ESTRUCTURA DE LOS GASTOS MUNDIALES EN INVESTIGACIÓN Y DESARROLLO}

\section{A) LA DISTRIBUCIÓN MUNDIAL DE LA I+D POR REGIONES Y PAÍSES}

Al inicio del siglo XXI, sobre un PBI mundial de 50.838 millardos de euros, el 1,6\%, es decir, casi 800 millardos, se invierten en I+D. El $91 \%$ de ese monto se concentra en tres

\footnotetext{
(5) Editado Paris: Económica, 2007.

(6) A saber: África del Sur, Argentina, China, Chipre, Eslovenia, Estonia, Israel, Letonia, Lituania, Malta, Rumania, Rusia, Singapur y Taiwán.

(7) En consecuencia, no debe olvidarse que la Economía del Saber también comprende las producciones del espíritu humano, es decir, todas aquellas que son del dominio del Derecho de Autor, recursos e ingresos que no son contabilizados en las cuentas de la OCDE ni en el Informe del OST.
} 
Regiones, a saber, América del $\operatorname{Norte}^{(8)}(36,1 \%), \operatorname{Asia}^{(9)}(30,6 \%)$, y la Unión Europea ${ }^{(10)}$ $(24,3 \%)$. Al interior de esas regiones, tres Estados conforman la cúspide del jerarquizado orden tecnológico mundial que se avizora para la economía mundial y el Sistema Internacional del siglo XXI: los EEUU (36\%), la UE (26\%) y el Japón (14\%). Es en estos tres actores de las relaciones económicas internacionales, en donde vive el $14 \%$ de la población mundial, y en donde se concentra el $76 \%$ de la inversión mundial en I+D.

En el período 1993 a 2003, en los países miembros de la OCDE, la I+D se multiplicó casi por dos, pero en los tres Estados líderes, la I+D, como parte de sus PBI, varió negativamente más en la UE $(-3,7 \%)$, que en el Japón $(-2,8 \%)$ y en los EEUU $(-1,5 \%)$. En el año 2004, la inversión en I+D, como parte de sus respectivos PBI, fue de 3,5\% en el Japón, de 2,6\% en los EEUU y de $1,8 \%$ en la UE.

Considerando: a) la parte que representa el capitalismo de EEUU en el total de la I+D mundial (35\%); b) el hecho de haber sido el que sufrió la menor variación negativa en el período analizado; y, c) el poseer una buena relación I+D sobre PBI, los EEUU aparecen con una importante ventaja relativa sobre sus socios/competidores en la Economía del Conocimiento.

\section{B) Los RECURSOS HUMANOS EN I+D, POR REGIÓN Y POR PAÍSES}

Observando y analizando las capacidades científicas y técnicas propias a la $\mathrm{I}+\mathrm{D}$ (cantidad de estudiantes inscriptos en la enseñanza superior y en las disciplinas "duras", la cantidad de profesores/investigadores a tiempo completo - ITC - en la producción/ difusión de la I+D), el dominio de la tríada vuelve a revelarse de manera implacable. En 2003, de los 5,5 millones de los ITC censados por el OST, Europa posee el 34,4\% (1.880.000), Asia el 34,2\% (1.870.000), América del Norte el 26,5\% (1.450.000), América Latina el 3,0\% (163.000), Australia-Nueva Zelanda el 1,6\% (89.000), y África el 0,3\% (15.000); al interior de las mencionadas regiones, los EEUU lideran con el 24,9\%, luego sigue la UE con el $21,6 \%$, la China con el 15,8\% y el Japón con el 12,4\%.

En los EEUU y en el Japón, los ITC trabajan fundamentalmente en el sector privado (80 y 70\% respectivamente), mientras que en la UE se distribuyen casi en partes iguales entre el sector público y el privado. En el período 1993-2003, la cantidad de ITC aumentó en un $18 \%$ en la UE, tres veces más que en los EEUU y seis veces más que en el Japón.

En el año 2004, la UE logró alcanzar la cifra de 91.052 doctores, dos veces más que los EEUU y seis veces más que el Japón; aunque en el período 1999-2004, el número de doctores en el Japón aumentó un 38\%, contra 20\% de la UE y 5\% en los EEUU.

Finalmente, en lo que concierne a la cantidad de estudiantes en estudios superiores, la UE y los EEUU registran la misma cantidad: casi 17 millones, mientras que el Japón sólo cuenta con 4 millones. Por otra parte, también es en la UE donde los estudiantes extranjeros realizan sus estudios superiores (casi 710.000 en el 2004), luego son los EEUU y el Japón. Por nacionalidad, los estudiantes chinos prevalecen claramente en el total de los estudiantes extranjeros, tanto en la UE como en los EEUU.

\footnotetext{
(8) Para la nomenclatura del OST, la Región comprende a los Estados Unidos y el Canadá

(9) Es decir, Japón, China, India, Corea del Sur, Singapur y Taiwán.

(10) Con 25 Estados miembros.
} 


\section{C) LA PRODUCCIÓN TECNOLÓGICA MEDIDA POR PATENTES DE INUENCIÓN}

Una invención que logra ser registrada, confiere a su titular un derecho exclusivo de explotación económica sobre un territorio determinado y por un período de tiempo, también estrictamente delimitado. A cambio, el productor-inventor debe entre otras obligaciones, hacer pública su invención. El registro de las patentes, es uno de los indicadores más comúnmente utilizados para medir la capacidad creativa del espíritu humano en materia científica técnica con aplicación industrial.

Existen actualmente, tres grandes sistemas de registro de la propiedad intelectual industrial: el sistema europeo, el sistema americano y el patent cooperation treaty (PCT).

En el 2004, el 63\% de las demandas de patentes en el sistema europeo fueron realizadas por los EEUU (30\%), el Japón (17\%) y Alemania (16\%); a su vez, en el sistema americano, el $77 \%$ de las demandas de patentes efectuadas fueron lideradas por los EEUU $(47,6 \%)$, el Japón (22,9\%) y Alemania (7,2\%). En el período 1993-2003, en los dos sistemas, la variación fue negativa, excepto en el sistema europeo donde la demanda Japonesa aumentó de un 12\%.

Pero, la capacidad en la producción tecnológica no sólo se mide por el número de invenciones realizadas y registradas, o en la demanda efectuada, sino también, en la llamada densidad tecnológica, es decir, en la cantidad de demanda de patentes de una zona o de un país, dividido por el total de sus habitantes. Así, en el sistema europeo, según la densidad tecnológica, la jerarquía la posee Suiza (1.845), seguida de Israel (881), Japón (868), los EEUU (669), Singapur (365), Canadá (343), Taiwán (88), África del Sur (36), Rusia (17) y Brasil (6).

En el sistema americano, aparecen en el primer lugar los EEUU (1.045), después viene Taiwán (1.023), Suiza (748), Israel (646), Canadá (448), Singapur (427), Corea del Sur (35), África del Sur (10), Rusia (7) y Brasil (3).

De lo anteriormente expuesto, se puede asociar la evolución de la cantidad de estudiantes de nivel superior provenientes de Asia con el número de ITC y la densidad tecnológica, y observar la estructura del probable nuevo orden tecnológico mundial y la reconfiguración del Poder de las Regiones, de los Estados en la Economía de la Información: junto a la tríada, emergen Israel, Suiza, Singapur y Taiwán, quedando relativamente en forma periférica, África del Sur, Rusia y Brasil, única economía latinoamericana que aparece casi siempre en el décimo lugar, en cualquier indicador que sobre desarrollo tecnológico se realice a partir de la nomenclatura de los países miembros de la OCDE.

Sobre los viejos y los nuevos actores y su peso relativo en el nuevo orden tecnológico mundial, volveremos al final de este trabajo. Veamos ahora, brevemente, la especialización y la división tecnológica en la tríada a través del llamado índice de especialización ${ }^{(11)}$, el cual permite visualizar la estrategia económica e industrial de un país o de una empresa (polivalencia o selectividad) así como, la división internacional de la producción y del comercio mundial sobre la cual se asientan las relaciones económicas internacionales y la economía mundial, en lo relativo a la Economía del Conocimiento.

(11) Es decir, el que mide la participación de un país en un dominio tecnológico específico, divida por la participación de ese mismo país en todos los dominios tecnológicos. 
En forma resumida, tanto en el sistema europeo como en el sistema americano, la Economía de la Información norteamericana muestra una fuerte especialización en ramas como la electrónica, la electricidad y sobre todo en el complejo fármaco-biotecnológico e instrumentos afines. A su vez, la UE se destaca en aquellas ramas donde los EEUU son menos especializados: máquinas, mecánica y transporte, procedimientos industriales, y en los artículos de los hogares. El Japón, en los dos sistemas viene en tercer lugar, fuertemente especializado en electrónica y electricidad, y en materiales químicos y muestra retraso con los EEUU y la UE en todas las otras ramas industriales ya mencionadas.

\section{D) LA PRODUCCIÓN CIENTÍFICA MEDIDA EN PUBLICACIONES}

En el año 2004, el 90\% de la producción científica mundial se concentra, por Regiones, en la UE (40\%), en América del Norte (30\%) y en Asia (20\%). Por Estados, casi un tercio de las publicaciones científicas mundiales son realizadas por los EEUU; lejos, le sigue el Japón $(8,5 \%)$, la China $(5,2 \%)$, Canadá $(3,3 \%)$, Rusia $(2,4 \%)$, India $(2,3 \%)$, Corea del Sur $(2,2 \%)$, Brasil y Taiwán $(1,4 \%)$, Suiza $(1,2 \%)$, y Singapur (1,5\%). En esos países, se concentra más de la mitad de las publicaciones científicas mundiales. Pero el poder tecnológico de una empresa, de un Estado, o de una Región, no sólo es visible y cuantificable por la parte y o la evolución que realiza sobre el total de las publicaciones científicas mundiales, sino también, y fundamentalmente, por la cantidad de veces que una invención publicada es citada en el mundo académico/científico. Para ello, existe el llamado índice de impacto ${ }^{(12)}$. Según este indicador, en 2004, los EEUU aparecen en primer lugar con un valor de este índice de 1,49, seguido de Suiza $(1,41)$, Canadá $(1,05)$, UE $(0,99)$, Israel $(0,95)$, Australia-Nueva Zelanda $(0,90)$, Japón $(0,86)$, Singapur y África del Sur $(0,63)$, y Corea del Sur $(0,56)$.

\begin{tabular}{|c|c|c|c|c|c|}
\hline \multicolumn{6}{|c|}{ LOS DIEZ ESTADOS MÁS PODEROSOS EN: } \\
\hline \multicolumn{3}{|c|}{$\begin{array}{c}\text { PBI } 2004 \\
\text { (millardos de dólares de 1995) }\end{array}$} & \multicolumn{3}{|c|}{\begin{tabular}{|c}
$\begin{array}{c}\text { En gastos en I+D en su PBI } 2003 \\
(\text { millardos de } € \text { en \%) }\end{array}$ \\
\end{tabular}} \\
\hline 1 & EEUU & 9.981 & 1 & Israel & 4,48 \\
\hline 2 & China & 6.576 & 2 & Japón & 3,15 \\
\hline 3 & Japón & 3.373 & 3 & EEUU & 2,68 \\
\hline 4 & India & 2.886 & 4 & Corea del Sur & 2,63 \\
\hline$\overline{5}$ & Alemania & 1.988 & 5 & Suiza & 2,57 \\
\hline 6 & Reino Unido & 1.484 & 6 & Taiwán & 2,45 \\
\hline 7 & Francia & 1.468 & 7 & Canadá & 1,95 \\
\hline 8 & Rusia & 1.453 & $y^{8}$ & Unión Europea & 1,80 \\
\hline 9 & Italia & 1.352 & 9 & China & 1,31 \\
\hline \multirow[t]{2}{*}{10} & Brasil & 1.259 & 10 & Rusia & 1,29 \\
\hline & & & 11 & Brasil & 0,95 \\
\hline
\end{tabular}

Fuente: Elaborado con datos de l'Économie Mondiale 2006. La Découverte, Collection Repère. Paris: CEPII, 2006. Fuente: Elaborado por el autor con datos de Indicateurs de Sciences et de Technologies. OST. Paris: Economica, 2007. p. 341

(12) Se define como la cantidad de citaciones bibliográficas de un texto en un determinado dominio tecnológico sobre el número total de publicaciones de ese dominio. 


\section{A Modo de Conclusión}

Considerando las informaciones fácticas enunciadas en el Informe del OST, se puede elaborar un primer bosquejo de la jerarquía tecnológica propia al capitalismo "tecnoinformacional" que marcará la economía mundial y las relaciones económicas internacionales en el siglo XXI.

En la Economía del Conocimiento, el Poder tecnológico está controlado por la tríada; los EEUU y el Japón logran conservar posiciones de privilegio entre los diez Estados más poderosos tecnológicamente, en cambio la UE pierde importantes posiciones, apareciendo en el octavo lugar (vide supra Cuadro).

$\mathrm{Al}$ interior de la tríada (EEUU, UE y Japón), las distancias tecnológicas, sean tanto en la densidad científica como en el índice de especialización, parecen haberse consolidado después del año 2004. En el último trienio, según el Monitor sobre la Inversión Industrial en $I+D$, publicado por la Comisión Europea (Bilan du Monde, 2008. p. 36), en agosto de 2007, las empresas europeas incrementaron sus inversiones en $\mathrm{I}+\mathrm{D}$ en un $10 \%$, mientras que las norteamericanas los hicieron en un 13\%, sobre el total de la OCDE. Las empresas de los EEUU lo hicieron fundamentalmente en los sectores donde son líderes (el complejo fármaco biotecnológico, las tecnologías de la información y la industria química). El mismo Informe confirma que la brecha tecnológica, en desmedro de la UE, ha aumentado debido a que las economías de la UE no han logrado alcanzar el objetivo que se habían fijado en Lisboa, de llegar a un $3 \%$ de su PBI en I+D.

A su vez, en la cima de la jerarquía de la Economía del Conocimiento, aparece en primer lugar el Estado de Israel, liderando el grupo de los Estados más poderosos en I+D, junto a EEUU y Japón (¿la nueva tríada tecnológica?). Surgen, en posición de privilegio, Corea del Sur, Suiza, Taiwán, Canadá, China y Rusia. En las próximas décadas se corroborará, si esta nueva jerarquía en la Economía del Conocimiento, refleja la reconfiguración y el desplazamiento de los nuevos centros de Poder tecnológico en el Espacio Económico Mundial

Prospectivamente podríamos pensar, al menos, en dos escenarios posibles concernientes al Orden Tecnológico del siglo XXI:

a) En uno de ellos, se puede estar asistiendo a un cambio en la cúspide de la economía mundial por el cual, junto a los viejos capitalismos de la Primera y la Segunda Revolución Industrial, emergen nuevas economías y nuevos Estados, conformándose una nueva jerarquía propia a la Economía del Conocimiento. En otros términos, y solamente a título ilustrativo, la economía mundial y el sistema internacional sufren un proceso relativamente similar al que conocieron en el período 1880-1918, cuando Japón y Alemania desafiaron el "Orden Europeo" y la Pax Británica. ¿El orden anglo-ruso-norteamericano (1870-1918 y 1918-1989) estaría siendo sustituido por el emergente orden euro-norteamericano-asiático?

b) En el otro escenario, no excluyente del anterior, el notable e intenso proceso de desarrollo capitalista de las últimas cuatro décadas en Asia puede interpretarse como el bosquejo de la división tecnológica mundial de la economía de la información. En 
dicha división, los viejos capitalismos occidentales conservan en sus territorios y en sus empresas el Poder, a través de los derechos de propiedad intelectual, del control oligopólicos de los circuitos comerciales, del establecimiento de las normas internacionales de comercio (OMC y G8), y, asientan la producción material en la periferia asiática, mediante la internacionalización/ deslocalización/ flexibilización de la producción.

En agosto de 2007, la OCDE publicó un documento sobre la Innovación en China (Bilan du Monde, 2008. p. 36), allí donde el capitalismo bate récords en materia de crecimiento, en las últimas cuatro décadas. Según los investigadores de la OCDE, la notable progresión de la economía china en el comercio mundial, se materializa a través de las ramas industriales que manifiestan mayor intensidad tecnológica; es en esas ramas donde predominan los sistemas de subcontratación, la tercerización y la flexibilización, es decir en otros términos, donde se ha instaurado la nueva relación social de producción propia al capitalismo de la información, la relación social de servicio.

Por otra parte, según el mismo documento de la OCDE, a pesar del aumento considerable del número de investigadores que conoce China, la productividad de los mismos sería muy baja, medida en función del número de publicaciones científicas y la cantidad de invenciones patentadas. Finalmente, el último trabajo de la OCDE pone en evidencia que los investigadores chinos trabajan, mayoritariamente, en los laboratorios de las empresas extranjeras que han deslocalizado la producción del conocimiento.

Mientras que la economía china ha alcanzado el segundo lugar en el PBI mundial (sumatoria del valor monetario de los bienes de uso final), aparece, sin embargo, en el noveno lugar de los diez Estados que invierten más en la Economía del Conocimiento.

Como fue anunciado al principio de este trabajo, las reflexiones sobre un Nuevo Orden Tecnológico propio al siglo XXI, no han hecho más que comenzar.

\section{REFERENCIAS BIBLIOGRÁFICAS}

AGAnBEGUiAn, Abel G. Pereströ̈ka. Le double défi soviétique. Paris: Economica, 1987.

ARCE, Gustavo. El capitalismo de la sociedad de la información. La Sociedad de la Información. Aspectos económicos, jurídicos y políticos de la nueva economía. Montevidéo: FCU, 2001.

BEAUD, Michel. Le Basculement du monde. Paris: La Découverte, 1997.

BILAN DU MONDE. L'Atlas de 174 pays. Paris: Le Monde, 2007.

. L'atlas de 174 pays. Paris: Le Monde, Hors Série, 2008.

COMISION ECONOMICA PARA AMERICA LATINA. CEPAL. Objetivos de desarrollo del milenio: una mirada desde América Latina y el Caribe. Informe Anual, ago. 2005.

Políticas públicas para el desarrollo de sociedades de información en América Latina y el Caribe. Informe Anual, jun. 2005.

COMISIÓN EUROPEA. La Unión Europea, América Latina y el Caribe: una asociación estratégica. Oficina de Publicaciones, 2005. Disponible en: <http://publications.eu.int $>$. 
CONFERENCIA DE LAS NACIONES UNIDAS SOBRE COMERCIO Y DESARROLLO. CNUCD. Informe sobre las inversiones en el mundo. Informes Anuales.

. Informe sobre el comercio y el desarrollo. Panorama general. CONFERENCIA DE LAS NACIONES UNIDAS SOBRE COMERCIO Y DESARROLLO. CNUCD. Informe sobre la economía de la información, 2005.

Informe sobre la economía de la información 2007-2008. El nuevo paradigma de las TIC. Panorama General.

DUVAL, Guillaume. L'entreprise efficace à l'heure de Swatch et McDonald's. La seconde vie du taylorisme. Paris: La Découverte \& Syros, 1998.

GADREY, Jean. L'économie des services. Paris: La Découverte, Collection Repères, 1996.

NACIONES UNIDAS. Desarrollo humano en Uruguay. 2005. Disponible en: < http://www.presidencia. gub.uy>.

Informe sobre el desarrollo humano. Anuales, PNUD, Disponible en: <http://www.pnud.org>. ORGANIZACIÓN DE NACIONES UNIDAS PARA EL DESARROLLO INDUSTRIAL. ONUDI. International year book of industrial statistic. Vienna, 2007.

ORGANIZACIÓN MUNDIAL DEL COMERCIO. OMC. Estadísticas del comercio mundial. Informes Anuales. Disponible en: <http://www.omc.org $>$.

RIFKIN, Jeremy. L'Age de L'Accès. La révolution de la nouvelle économie. Paris: La Découverte, 2000 .

ROSEMBAUM, Jorge; CASTELLO, Alejandro. Régimen jurídico de la subcontratación e intermediación laboral. Montevideo: FCU, 2007. 